\title{
Introduction to the Retransmission Scheme Under Cooperative Diversity in Wireless Networks
}

\author{
Yao-Liang Chung ${ }^{1}$ and Zsehong Tsai ${ }^{2}$ \\ Graduate Institute of Communication Engineering, \\ National Taiwan UniversityTaipei \\ Taiwan, R.O.C.
}

\section{Introduction}

As implied by the word "Cooperative Diversity (CD)," mobile users in a multi-user environment can share their antennas in a manner that creates a virtual Multiple-Input Multiple-Output (MIMO) system, which can be conceptually viewed as a multichannel transmission environment in the network layer, to achieve individual or common purposes of those users. By employing CD for transmissions, the quality and reliability of users' data in wireless networks can thus be improved, mainly owning to the reason that the effect of wireless channel fading can be reduced. In this chapter, we aim to introduce existing representative retransmission schemes under various environments and further present a novel packet retransmission scheme for Quality-of-Service (QoS)-constrained applications in a general CD environment.

Transmit diversity of MIMO systems is an important technique which can bring significant gain to wireless systems with multiple transmit antennas. This technique is clearly advantageous to be employed on a cellular base station; however, it may not be practical for other scenarios. To be more specific, due to size, cost, or hardware limitations, small handsets/cellular phones may not be able to support certain types of multiple transmit antennas. For example, the size of an antenna must be several times the wavelength of the carrier frequency. Therefore, the use of multiple antennas is not an attractive way to achieve the transmit/receiving diversity in small handsets/cellular phones. To overcome such a naturally fundamental problem, $\mathrm{CD}$ is in nature an effective strategy to allow a singleantenna mobile device to achieve the benefit of MIMO systems with the help of cooperative mobile devices.

$\mathrm{CD}$, which is a form of spatial diversity, is through cooperating users' (usually called partners) relaying signals to the destination. This technique is achieved without the use of additional antennas of any user. That is to say, the antennas of the sender and partners together form a multiple-transmit antenna situation. Basically, the relay mechanism can be decode-and-forward or amplify-and-forward. Moreover, CD is an emerging and powerful technique that can mitigate fading and improve robustness to interference in wireless environments. Thus, CD becomes a promising candidate for emulating MIMO systems. 
Recently, many research groups have turned their attention to the CD-related topics. Individual aspects of these problems have been considered, for example, in various papers [1-4]. In [1], Mahinthan et al. proposed a Quadrature Signaling (QS) mechanism in the CD system for transmissions. CD transmissions considering issues related to power allocation algorithms were explored by Mahinthan et al. in [2-3]. In [4], Chen et al. exploited that the use of space-time block coding in the multi-user $C D$ to improve the performance of the transmission in wireless local area networks. Other abounding literature survey and investigation regarding the issue related to $C D$ including principles and applications can be referred to Ray Liu et al. in [5] and Fitzek et al. in [6], respectively. Recently, a simple method to evaluate the performance of complex networks under CD using sampling property of a delta function was proposed by Jang in [7].

However, because of fundamental physical characteristics of wireless channels, data packets often cannot be delivered to the destination successfully. As a result, the design focusing on the efficient retransmission scheme under such a CD environment still plays a highly crucial role. Due to the evolution of the communication technology, most packet retransmission schemes under $C D$ in literatures were based on the rich results from those retransmission schemes on point-to-point transmissions. Thus, we will first provide an overview of retransmission schemes on point-to-point transmissions, and then, investigate the issue on the retransmission scheme under the CD environment.

While there have been many papers exploring various retransmission schemes in the CD environment, there were no elaborations on the issue considering the time constraint for delay-sensitive services. Consequently, in such a CD environment their throughput formulas did not reflect the effective throughput (goodput) that must satisfy the typical delay constraints of streaming-type or real-time multimedia flows. Motivated by the above point, we therefore pay our attention to design a novel fast packet retransmission scheme to be employed in a general CD environment for delay-sensitive flows as a case study.

The rest of this chapter is organized as follows. A survey of various retransmission schemes is included in Section 2. Next, Section 3 proposes a novel fast packet retransmission scheme in a general CD environment for delay-sensitive applications as a case study. Section 4 makes a summary of this chapter and suggests the future work of interest. Finally, the list of references is provided in the end.

\section{A survey of various retransmission schemes}

The traditional retransmission scheme designed to combat the loss of transmission data for single-radio single-channel environments was first introduced by Lin et al. in [8]. Thanks to the advances of multi-band radio technologies, for many broadband wireless systems and short range communication networks, there may be many communication channels available to use. Consequently, it is natural to arrange the link layer packets to be transmitted over multiple channels to boost bandwidth. Issues regarding how to design and analyze the multi-channel transmission schemes have recently become an important research direction. A vast amount of research groups have thus started to pay attention to related topics. Individual aspects of these problems have been separately considered in many related papers [9-18]. Most literatures in this area can be conceptually categorized into 2 research directions; one is the single-radio multi-channel transmission discussed in [9-11], 
and the other direction is the multi-radio multi-channel transmission discussed in [12-18]. Meanwhile, for the single-radio single-channel cases, the performance results of the Automatic-Repeat-reQuest (ARQ) retransmission schemes based on Markov analysis were available in [19-22], and optimized in both the power and the packet drop probability aspects respectively were recently studied in [23]. These studies [9-23] together provide a basis for the analysis and comparison of the multi-channel transmission. Furthermore, various kinds of ARQ and Hybrid ARQ schemes designed to be employed for CD environments were explored in [24-27]. The design approaches of these related research works are elaborated as follows.

In [9], a protocol was proposed to enable hosts to utilize multiple channels by switching channels dynamically, and their simulation showed that the effective throughput was improved, especially when the network was highly congested. Centralized and distributed algorithms to perform efficient channel assignments in component-based approach were proposed and implemented in [10]. A joint multi-channel and multi-path control protocol was proposed in [11], where it combined multi-channel link layer with multi-path routing, and simulations showed that the scheme can improve the throughput significantly.

The uses of switchable interfaces and multi-channel routing were proposed in [12], and simulation results showed that the throughput in a wireless ad hoc network can be improved. In [13], a hybrid channel assignment scheme was modeled into an integer linear programming formulation, and an approximation method for simplification was also studied. In the ad hoc network related areas, [14] maximized the network throughput subject to fairness requirements using the proposed wireless network coding schemes for a variety of multi-radio multi-channel environments with different routing strategies.

Several works focusing on the ARQ schemes in multi-radio multi-channel transmissions have been discussed in [15-18]. Formulas of the link layer throughput for the Stop-and-Wait (SW), the Go-Back-N (GBN) or the Selective-Repeat (SR) schemes were derived under different assumptions of channel characteristics. The multi-channel SR ARQ scheme in [15] assumed equal transmission rate and allowed the transmitter dynamically to assign the retransmission link packet to the channel using the link packet error probability as the selection criterion, and retransmissions can continue till its success. In [16], both throughput and delay performances of the multi-channel SW, GBN, and SR ARQ schemes were investigated and validated via simulations, while all channels were assumed statistically independent and identical. Throughput analysis of the multi-channel SW, GBN, and SR ARQ schemes were generalized in [17], where the generalization took the form of packet-tochannel assignment rules, and radio channels can be with different transmission rates and different link packet error probabilities. Two fast ARQ/HARQ packet retransmission schemes have been proposed to transport delay-sensitive flows in a multi-radio multichannel environment in [18], where they can incorporate various retransmission policies, which are adjusted by the channel signal-to noise ratio (SNR) and the APDU size.

Closed form equations of the service data unit delay under the SR ARQ scheme were successfully derived and validated via simulation in [19]. An exact Markov model proposed to evaluate the delay statistics of the link packet for the SR ARQ scheme was available in [20]. The queueing models using dynamic link adaptation for the GBN and the SR ARQ schemes were formulated in [21], where the exact queue length and the delay statistics were 
obtained. For HARQ schemes, a Markov model was presented to analyze the SR truncated type II HARQ scheme employing Reed Solomon linear erasure block codes in [22], where the link packet throughput, error probability, and delay performance were analyzed. In [23], a suboptimal root-finding solution was developed to solve the exhaustive search for the optimization problem formulated based on the incremental-redundancy HARQ scheme.

The delay performance of several truncated ARQ and HARQ schemes in a CD environment under the assumption of Poisson arriving packets were evaluated in detail by Boujemâa in [24]. An analytical model to quantify end-to-end performances for a CD ARQ scheme in a cluster-based multi-hop wireless network was proposed by Le et al. in [25]. Markov models developed to evaluate the CD system were also investigated by Mahinthan et al. in [26] and by Issariyakul et al. in [27], respectively.

While papers [24-27] have widely explored various ARQ/HARQ schemes in the CD environments, the issues regarding time constraints for delay-sensitive flows were not addressed and elaborated on. Therefore, their throughput formulas did not reflect the effective throughput that must satisfy the typical delay constraints of streaming-type or realtime multimedia flows in such an environment.

Due to the aforementioned reasons, we herein propose a novel fast packet retransmission scheme, where a new approach of retransmission strategy is designed and appropriately combines the encoding/decoding mechanism presented in [18], in such a CD environment for delay-sensitive flows as a case study. In the proposed scheme, there are 2 retransmission policies that can be employed adaptively according to both the channel quality and the Application layer Protocol Data Unit (APDU) size. The retransmission is designed to be allowed only one time. Here, APDU flows in the sender are further assumed to always have a link packet ready for transmission. As a result, it is not much meaningful to analyze the packet delay involving the queueing analysis. In this paper, we only focus on the complete throughput analysis to gain the main insight of optimizing the number of channels for retransmission between the 2 proposed retransmission policies under such the CD environment. All of the derived formulas are then verified via simulations. The effective throughput of our proposed scheme is shown better than that of other CD retransmission schemes (such as [26]) and non-CD retransmission schemes.

\section{Case study: On the effective throughput gain of cooperative diversity with a fast retransmission scheme for delay-sensitive flows [33-34]}

\subsection{System description}

\subsubsection{Cooperative diversity system}

A general CD system model composing of a sender, a partner, and a receiver is considered, as shown in Fig. 1, where two cooperative users (i.e., sender and partner) transmit their information to the same destination (i.e., receiver). It is assumed that each user' device in this system only has one radio transceiver. Additionally, Orthogonal Frequency Division Multiplexing (OFDM) is employed as the underlying transmission technique.

In the present system, channels among sender, partner and receiver are modeled as nonidentical but independent Nakagami- $m$ slow-fading channels corrupted by additive white Gaussian noise. The fading channels and the noise are assumed to be independent of each other. 


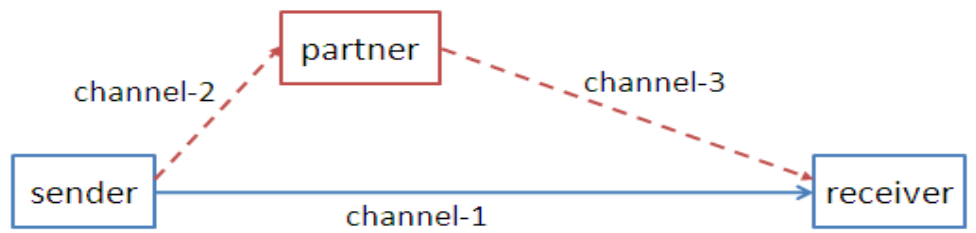

Fig. 1. System model for two cooperative users (sender and partner) transmission.

Generally speaking, for the transmission of flows of the sender, application layer flows are composed of APDUs. We assume that an APDU consists of $s$ link packets. Each link packet will be encoded (described in detail in Section 3.1.3) in sequence for transmission. Here, APDU flows in the sender are assumed to always have a link packet ready for transmission.

For the convenience of the following analysis, channels that between sender and destination, between sender and partner, and between partner and destination are denoted as channel- $j$, $j=1,2,3$, respectively.

Last, but not least, we herein choose to employ only 1 partner for study since the significant improvement of the overall system performance with $\mathrm{CD}$ is usually owing to the contribution of the best partner [3].

\subsubsection{Principles of fast retransmission strategy}

The design philosophy of the retransmission strategy is to improve the application layer throughput while the effective control of the transmission delay is also assured. We assume that the underlying coding scheme is HARQ and that if a packet is retransmitted, then only its complementary packet is sent.

The packet retransmission strategy can be described via the following 4 principles:

- A link packet will be duplicated a copy in the sender buffer before its first transmission. When the sender begins to transmit this link packet, it will be broadcasted to the receiver and the partner.

- When an original link packet is transmitted to the receiver, an acknowledgment (ACK) or Negative ACK (NACK) packet (assumed error-free) will be sent to both the sender and the partner.

- There are 2 retransmission policies designed for the retransmission, indexed as policy_k: for $k=0,1$. If a NACK is received, a complementary link packet will be retransmitted only one time via the partner (indexed as policy_0) or via both the sender and the partner (indexed as policy_1).

- The best retransmission policy is selected based upon both the APDU length and the expected long term link packet error probability among sender, partner and receiver, using the average application throughput as the performance objective. Under different APDU sizes and different link packet error probabilities, the corresponding best retransmission policy can be different.

Since an OFDM system is assumed, we assume each channel only uses a subset of OFDM subchannels. For the above retransmission strategy, note that both channel-1 and channel-2 
are with orthogonal subchannel set 1 , while channel-3 is with orthogonal subchannel set 2 . The intersection of subchannel set 1 and set 2 is arranged to be an empty set; therefore, for the receiver, the signals from channel- 1 and channel-3 will not interfere with each other.

Typical retransmission operations under policy_0 and policy_1 are illustrated in Fig. 2 and Fig. 3, respectively. Also, we assume that the delay threshold of the considered delaysensitive APDU is set equal to the maximum of maximum delays for 2 policies. Notice that in this model the terminology delay only indicates the air-transmission delay component for the APDU under the proposed retransmission principle.

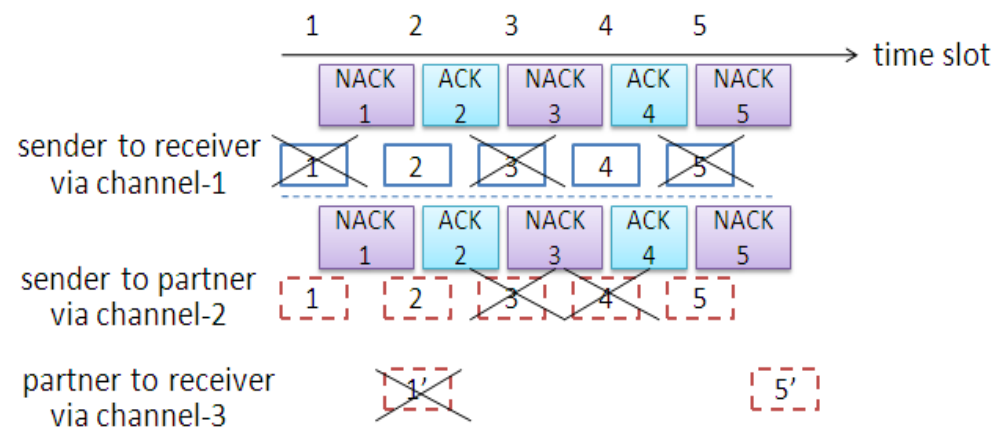

Fig. 2. A typical example of a fast HARQ with policy_0. When any original link packet is found failed at the receiver, only the partner will retransmit a complementary link packet if a link packet is successfully received by the partner. Link packet i' means the complementary packet of link packet $i$.

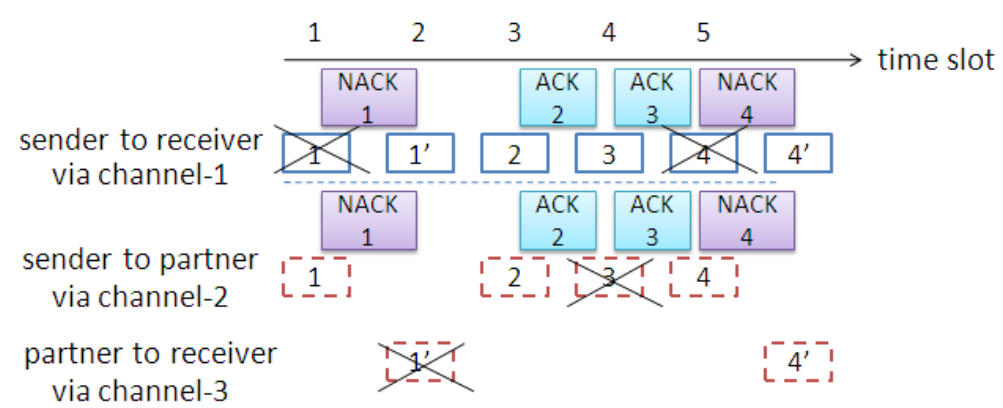

Fig. 3. A typical example of fast HARQ with policy_1. When any original link packet is found failed in the receiver, both the sender and the partner will retransmit a complementary link packet. Link packet $i^{\prime}$ is the complementary packet of link packet $i$.

\subsubsection{Cooperative diversity with fast HARQ scheme}

Two codes, a block code $C_{0}$ and a convolutional code $C_{1}$, are together used as the coding mechanism employed in each user (see [29], [36] for examples). For more detail description, $C_{1}$ is a rate- $1 / 2$ convolutional code with constraint length $c$ consisting of a $c$-stage shift 
register and two generator polynomials $G_{1}(x)$ and $G_{2}(x)$. This code $C_{1}$ is used as an inner code for error detection and correction. Next, the outer code $C_{0}$ is a high rate $(n-(c-1), n-(c-1)-r)$ block code used for error detection only, where $n$ is the length of each link packet that is transmitted in this scheme, and $r$ is the length of parity-check bits for error detection.

When an APDU arriving at the sender, there will be $s$ new information sequences $I_{i}(x)$, $1 \leq i \leq s$, each length $(n-(c-1)-r)$, generated in sequence. They are encoded into $J_{i}(x)$ with $C_{0}$ and then encoded into $V_{i}(x)=J_{i}(x) G_{1}(x)$ with $C_{1}$ in sequence. Each of them will be broadcasted in sequence only one time to the partner and the receiver.

Let $\tilde{V}_{i}(x)$ and $\hat{V}_{i}(x)$ be the noisy versions of $V_{i}(x)$ arriving at the receiver and the partner, respectively. For the receiver, the syndrome of $\tilde{V}_{i}(x)$ is checked in two steps. In step_1, $\tilde{V}_{i}(x)$ is regarded as a noisy version of a codeword in the $(n, n-(c-1))$ shortened cyclic code generated by $G_{1}(x)$. In step_2, an estimate $\tilde{J}_{i}(x)$ of $J_{i}(x)$ is then checked in the high rate $(n-(c-1), n-(c-1)-r)$ block code. If the syndromes are all zero in any step, an estimate $\tilde{I}_{i}(x)$ of $I_{i}(x)$ is obtained and delivered to the receiving buffer, which is a buffer used for waiting other link packets back for an APDU. Subsequently, an ACK packet will be sent to the sender and the partner. However, if the aforementioned syndrome check in any step is not zero, $\tilde{V}_{i}(x)$ is stored in the receiver buffer, and a NACK packet will be transmitted to the partner and the sender for possible retransmission. For the partner, if $\hat{V}_{i}(x)$ is error-free, then either policy_0 or policy_1 is adopted; otherwise, it is directly dropped.

Under policy_0, if $\hat{V}_{i}(x)$ is error-free, $\hat{V}_{i}(x)$ will be decoded to $V_{i}(x)$ and then re-encode via $G_{2}(x)$ to $V_{i}^{\prime}(x)$. Thereafter, $V_{i}^{\prime}(x)$ is transmitted to the receiver. Let the noisy version of $V_{i}^{\prime}(x)$ be denoted as $\hat{V}_{i}^{\prime}(x) . \hat{V}_{i}^{\prime}(x)$ will be checked in the same way as in the first transmission. If $\hat{V}_{i}^{\prime}(x)$ is found failed, $\hat{V}_{i}^{\prime}(x)$ shall be combined with $\tilde{V}_{i}(x)$, to form a combined codeword, which is decoded by the Viterbi decoding. The result is checked in the high rate $(n-(c-1), n-(c-1)-r)$ block code. If the syndrome is zero, it is claimed as a correct result. Its information sequence is then estimated and delivered to the receiving buffer; otherwise, it is discarded and the retransmission for this link packet is stopped.

Under policy_1, a complementary link packet $V_{i}^{\prime}(x)$ via $G_{2}(x)$ of $V_{i}(x)$ will be transmitted from the sender to the receiver, and let the noisy version be denoted as $\tilde{V}_{i}(x)$. Meanwhile, if $\widehat{V}_{i}(x)$ is error-free, $\hat{V}_{i}(x)$ will be decoded to $V_{i}(x)$ and then re-encode via $G_{2}(x)$ to $V_{i}^{\prime}(x)$. Let the noisy version of $V_{i}^{\prime}(x)$ be denoted as $\hat{V}_{i}^{\prime}(x)$. Following that, $\tilde{V}_{i}(x)$ and $\hat{V}_{i}^{\prime}(x)$ will be checked via the two-step decoding procedure, respectively. If the syndrome of any one is zero, it is claimed as a correct result, its information sequence is then estimated and delivered to the receiving buffer; otherwise, $\tilde{V}_{i}(x)$ shall be combined with $\hat{V}_{i}^{\prime}(x)$ and $\tilde{V}_{i}(x)$, respectively, to form two combined codewords, which are decoded by the Viterbi decoding. If any result is successful, its information sequence is then estimated and delivered to the receiving buffer; otherwise, a new codeword, $\hat{V}_{i, m r c}(x)$, will be further generated based on the Maximal Ratio Combining (MRC) (see [31] for more details) technique via $\hat{V}_{i}^{\prime}(x)$ and $\tilde{V}_{i}(x)$. The syndrome of the new codeword is checked in the same concept of the two-step decoding procedure. If the result is successful, the estimated information sequence will be delivered to the receiving buffer; otherwise, it is discarded and the retransmission for this link packet is stopped. 


\subsection{Throughput analysis}

Performances of the application layer throughput for the present scheme will be first analyzed in detail in this Section. It is assumed that the time axis is partitioned into equal size slot. In each time slot, it is separated into two parts. That is to say, the main part of a time slot is used for link packets transmissions and the rest of the time slot is reserved for ACK/NACK packets transmissions. Here, the SNR is assumed staying constant in a time slot. In addition, the $M$-ary, $M=2^{b}$ where $b$ is even, the Quadrature Amplitude Modulation (QAM) scheme is assumed in the OFDM subchannels of the proposed model.

\subsubsection{Link packet error probability}

For $M$-ary QAM in Nakagami- $m$ slow-fading channels, the average BERs for channel- $j$, $j=1,2,3$, denoted as $\bar{\varepsilon}_{j}$, can be derived by

$$
\bar{\varepsilon}_{j}=\int_{0}^{\infty} p_{j}\left(\gamma_{j}\right) \varepsilon_{i n s, j}\left(\gamma_{j}\right) d \gamma_{j}, j=1,2,3,
$$

where, in channel- $j, \varepsilon_{i n s, j}\left(\gamma_{j}\right)$ is the instantaneous BER conditional on $\gamma_{j}$ for M-ary QAM, $\gamma_{j}$ is the instantaneous SNR per bit, $\gamma_{j}>0, p_{j}\left(\gamma_{j}\right)=m^{m} \gamma_{j}^{m-1} e^{-m \gamma_{j} / \bar{\gamma}_{j}} / \bar{\gamma}_{j}^{m} \Gamma(m)$ is the probability density function (pdf) of $\gamma_{j}$ in Nakagami- $m$ fading given in [28], $m \geq 1 / 2, \Gamma(\cdot)$ is the gamma function, and $\bar{\gamma}_{j}$ is the average SNR per bit. The instantaneous BER $\varepsilon_{i n s, j}\left(\gamma_{j}\right)$ was previously derived in [30], [32] as

$$
\begin{aligned}
& \varepsilon_{\text {ins }, j}\left(\gamma_{j}\right)= \\
& \frac{1}{\sqrt{M} \log _{2} \sqrt{M}} \sum_{z=1}^{\log _{2} \sqrt{M}} \sum_{t=0}^{f(z, M)}\left\{\operatorname{erfc}\left((2 t+1) \sqrt{g \gamma_{j}}\right) f(t, z, M)\right\}, j=1,2,3,
\end{aligned}
$$

where

$$
f(z, M)=\left(1-2^{-z}\right) \sqrt{M}-1,
$$

$g=3 \log _{2} M /(2 M-2)$,

$f(t, z, M)=(-1)^{\left\lfloor t 2^{z-1} / \sqrt{M}\right\rfloor}\left(2^{z-1}-\left\lfloor t 2^{z-1} / \sqrt{M}+1 / 2\right\rfloor\right)$, and $\operatorname{erfc}(\cdot)$ is the error function.

The average link packet error probability in a single transmission in channel- $j, j=1,2,3$, denoted as $\bar{P}_{j, e}$, can be given by

$$
\bar{P}_{j, e}=\int_{0}^{\infty} p_{j}\left(\gamma_{j}\right)\left(1-\left(1-\varepsilon_{i n s, j}\left(\gamma_{j}\right)\right)^{n}\right) d \gamma_{j}, j=1,2,3
$$

Furthermore, the average link packet error probability after the Viterbi decoding conditional on the event that both $\hat{V}_{i}^{\prime}(x)$ and $\tilde{V}_{i}(x)$ are corrupted, denoted as $\bar{P}_{f, 0}$, can be approximately by (see eq. (28) in [29])

$$
\bar{P}_{f, 0} \cong 1-\left(1-p_{b}\right)^{n-(c-1)},
$$


where $p_{b}$ is the corresponding bit error probability obtained via the Viterbi decoding. As shown in [29], $p_{b}$ is bounded by

$$
p_{b} \leq\left.\frac{1}{2} \frac{\partial T(X, Y)}{\partial Y}\right|_{X=2 \sqrt{\varepsilon^{\prime}\left(1-\varepsilon^{\prime}\right)}, Y=1},
$$

where $\varepsilon^{\prime}$, the upper bound of the conditional BERs given that the two-step (mentioned in Section 3.1.3) decoding syndromes in channel-1 and channel-3 are non-zero, is given by

$$
\varepsilon^{\prime}=\max \left\{\frac{\varepsilon_{1}}{1-\left(1-\varepsilon_{1}\right)^{n}}, \frac{\left(1-\varepsilon_{2}\right) \varepsilon_{3}}{1-\left(1-\left(1-\varepsilon_{2}\right) \varepsilon_{3}\right)^{n}}\right\},
$$

and $T(X, Y)$ is the generating function of the convolutional code. In addition, $\bar{P}_{f, 1}$, the average link packet error probability after the Viterbi decoding conditional on the event that both $\tilde{V}_{i}(x)$ and $\tilde{V}_{i}(x)$ are corrupted, can be given by (4)-(6) together with $\varepsilon$ ' replaced by $\varepsilon=\varepsilon_{1} /\left(1-\left(1-\varepsilon_{1}\right)^{n}\right)$, which is the conditional BER given that the two-step decoding syndrome in channel-1 is non-zero.

Last, but not least, the average link packet error probability after the MRC decoding, under policy_1, denoted as $\bar{P}_{m r c}$, can be given by

$$
\bar{P}_{m r c}=\int_{0}^{\infty} \tilde{p}\left(\gamma_{b}\right)\left(1-\left(1-\varepsilon_{m r c}\left(\gamma_{b}\right)\right)^{n}\right) d \gamma_{b}
$$

where $\gamma_{b}$ is the instantaneous SNR per bit at the output of the MRC decoder, $\tilde{p}\left(\gamma_{b}\right)$ represents the pdf of $\gamma_{b}, \gamma_{b}>0$, and $\varepsilon_{m r c}\left(\gamma_{b}\right)$ is the instantaneous BER conditional on $\gamma_{b}$ for $M$-ary QAM after the MRC decoding. According to [28], [30], $\tilde{p}\left(\gamma_{b}\right)=m^{2 m} \gamma_{b}^{2 m-1} e^{-m \gamma_{b} / \bar{\gamma}_{c}} / \bar{\gamma}_{c}^{2 m} \Gamma(2 m)$, where $\bar{\gamma}_{c}$ means the equivalent average SNR for each channel. The instantaneous BER $\varepsilon_{m r c}\left(\gamma_{b}\right)$ can be given by (2) with $\gamma_{j}$ replaced by $\gamma_{b}$.

\subsubsection{Throughput}

For the fast HARQ scheme with policy_0, the application layer throughput in APDU/slot, denoted as $T_{0}$, can be derived as

$$
T_{0}=\frac{1}{s}\left(1-\bar{P}_{1, e} \bar{P}_{2, e}-\bar{P}_{1, e}\left(1-\bar{P}_{2, e}\right) \bar{P}_{3, e} \bar{P}_{f, 0}\right)^{s}
$$

where $1 / s$ represents the average number of the APDUs transported per slot, and the second term indicated the success probability of an APDU transmission.

Next, for the fast HARQ scheme with policy_1, the application layer throughput in APDU/slot, denoted as $T_{1}$, can be derived as

$$
T_{1}=\frac{\alpha}{s}\left(1-\bar{P}_{1, e}^{2} \bar{P}_{2, e} \bar{P}_{f, 1}-\bar{P}_{1, e}^{2}\left(1-\bar{P}_{2, e}\right) \bar{P}_{3, e} \bar{P}_{f, 0} \bar{P}_{f, 1} \overline{\bar{P}}_{m r c}\right)^{s}
$$


where $\alpha / s$ represents the average number of the APDUs transported per slot, and similar to the concept in (8), the second term means the success probability of an APDU transmission. In (9), $\overline{\bar{P}}_{m r c}$ is the average link packet error probability after the MRC decoding conditional on $\hat{V}_{i}^{\prime}(x)$ and $\tilde{\tilde{V}}_{i}(x)$ all found failed. Since $\bar{P}_{m r c}$ is the unconditional probability of a link packet error after the MRC decoding and the result will be correct after the MRC decoding as long as there is at least a link packet that is correct, $\overline{\bar{P}}_{m r c}$ can be derived as $\overline{\bar{P}}_{m r c}=\bar{P}_{m r c} /\left(\bar{P}_{1, e}^{2}\left(1-\bar{P}_{2, e}\right) \bar{P}_{3, e}\right)$ by the definition of conditional probability [35]. Moreover, $\alpha$ in (9) can be obtained via the equality

$$
\bar{P}_{1, e} \alpha=1-\alpha,
$$

since the average number of retransmission link packets generated per slot should equal the average number of retransmission completed, after normalization.

Last, but not least, for delay-sensitive flows, the maximum air-transmission delay of an APDU allowed is usually subject to a specific QoS requirement. In this case, based on the similar derivation and argument in [18], one can appropriately tune the key parameter, namely, $s$, in the system to achieve the highest effective throughput under a given delay constraint.

\subsection{Analytical and simulation results}

In this section, the considered $C D$ environment with a sender, a partner, and a receiver remains the same as shown in Fig. 1. We assume that an APDU is composed of 5 link packets. A 16 QAM modulation scheme is adopted. The coding mechanism is referred to Section 3.1.3. Also, we set $r=6$ bytes, $c=9$, and $n=257$ bytes. The ACK/NACK packet size for ARQ related schemes is set equal to 25 bytes and for HARQ related schemes is set equal to 26 bytes. The link speed is set equal to $10 \mathrm{Mbps}$. Besides, excluding the errorcorrecting codes, the ratio of the additional header overhead associated with the lower layer protocols from the application one is set equal to 0.04 .

First, we will evaluate and compare the performance results among all schemes to see main potential insights of our proposed scheme by considering the ideal case that the channel between the sender and the partner is error-free. Next, we further investigate the impact on the system performance when there is an error probability on the channel between the sender and the partner.

\subsubsection{With an error-free channel-2}

For the fast retransmission scheme, based on (8)-(9), analytical results of application throughputs under $\bar{\varepsilon}_{1}$, with $\bar{P}_{3, e}=0.9$ and $\bar{P}_{3, e}=0.1$, in the Nakagami-3 slow-fading environment, are depicted in Fig. 4 and Fig. 5, respectively. In Fig. 4, it can be found that if $\bar{\varepsilon}_{1} \leq 0.4 \times 10^{-2}$, the optimal throughput can be achieved with only 1 channel for retransmission (via the partner); if $\bar{\varepsilon}_{1} \geq 0.4 \times 10^{-2}$, it can be achieved by parallel retransmissions via 2 channels (via both sender and partner). However, in Fig. 5, it is seen 
that the throughput of policy_0 is always better than that of policy_1. Because the average link packet error probability of channel-3 is small, the retransmission of duplicated link packet on channel-1 via policy_1 will waste bandwidth.

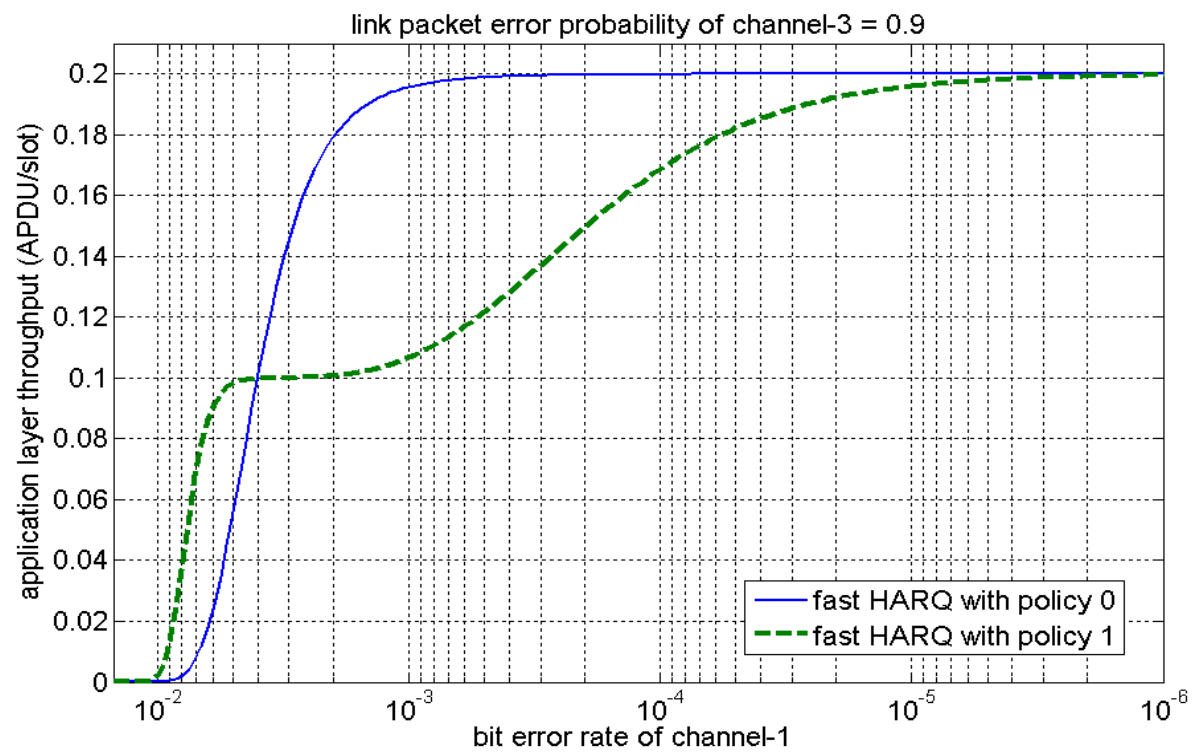

Fig. 4. Application throughputs under typical $\bar{\varepsilon}_{1}$ with $\bar{P}_{3, e}=0.9$ under a fast HARQ scheme.

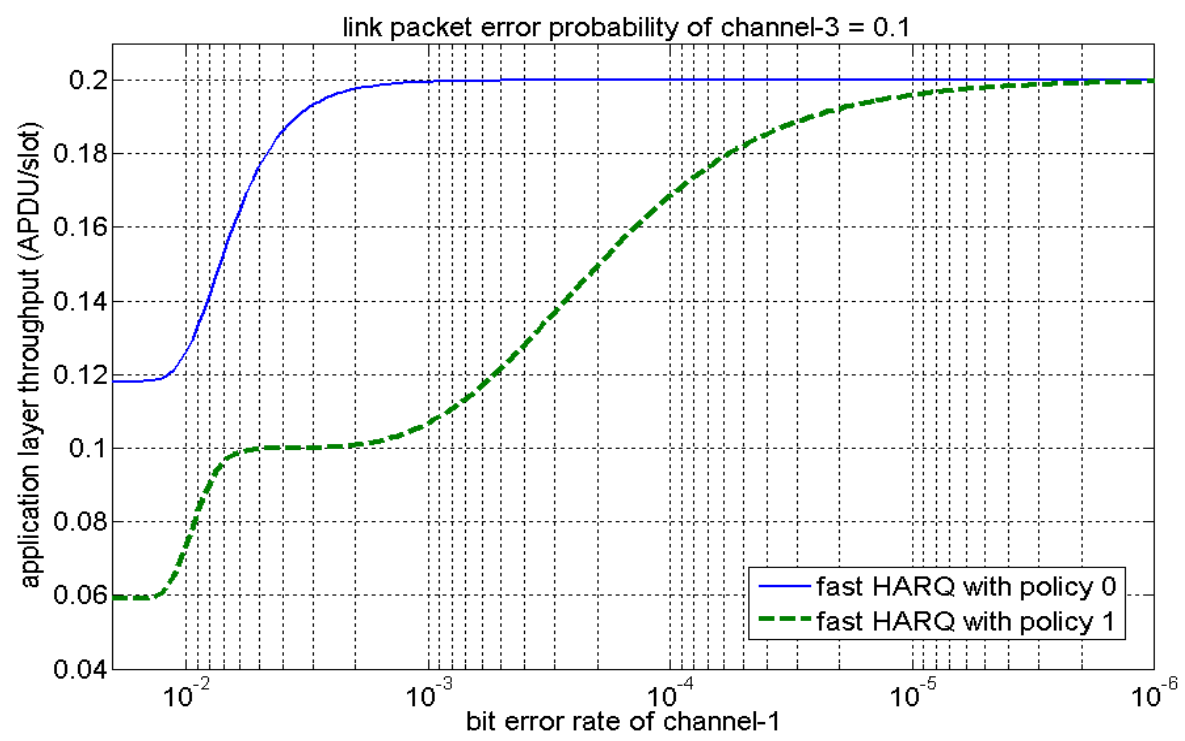

Fig. 5. Application throughputs under typical $\bar{\varepsilon}_{1}$ with $\bar{P}_{3, e}=0.1$ under a fast HARQ scheme. 
In what follows, we compare the throughput of our proposed scheme with that of the previous work [26] and non-CD HARQ scheme under $\bar{\varepsilon}_{1}$, with $\bar{\varepsilon}_{3}=10^{-3}$ in Nakagami-3 slow-fading channels, as shown in Fig. 6. Notice that in Fig. 6, the $C D$ with optimized fast $H A R Q$ scheme represents the case that the retransmission policy is adaptively adjusted to be optimal on the basis of the channel quality in the CD environment. For a fair comparison among all schemes, all throughput results are in bit/second, and other 2 schemes are modified to allow only 1 retransmission and time slots for those discarded retransmissions are then used for new transmissions. Notice due to this modification, their throughput formulas are modified versions of (9) with the unused parameters removed. In details, one should set $\bar{P}_{f, 1}=1$ and replace the parameter $n$ in (3) by $n-(c-1)$ for the scheme in [26], and set $\bar{P}_{2, e}=1$ for the non-CD HARQ scheme. With the help of Fig. 6, it can be found that better performance is achieved by the optimized fast HARQ scheme except when $\bar{\varepsilon}_{1}$ is extremely small due to the additional overhead of the HARQ.

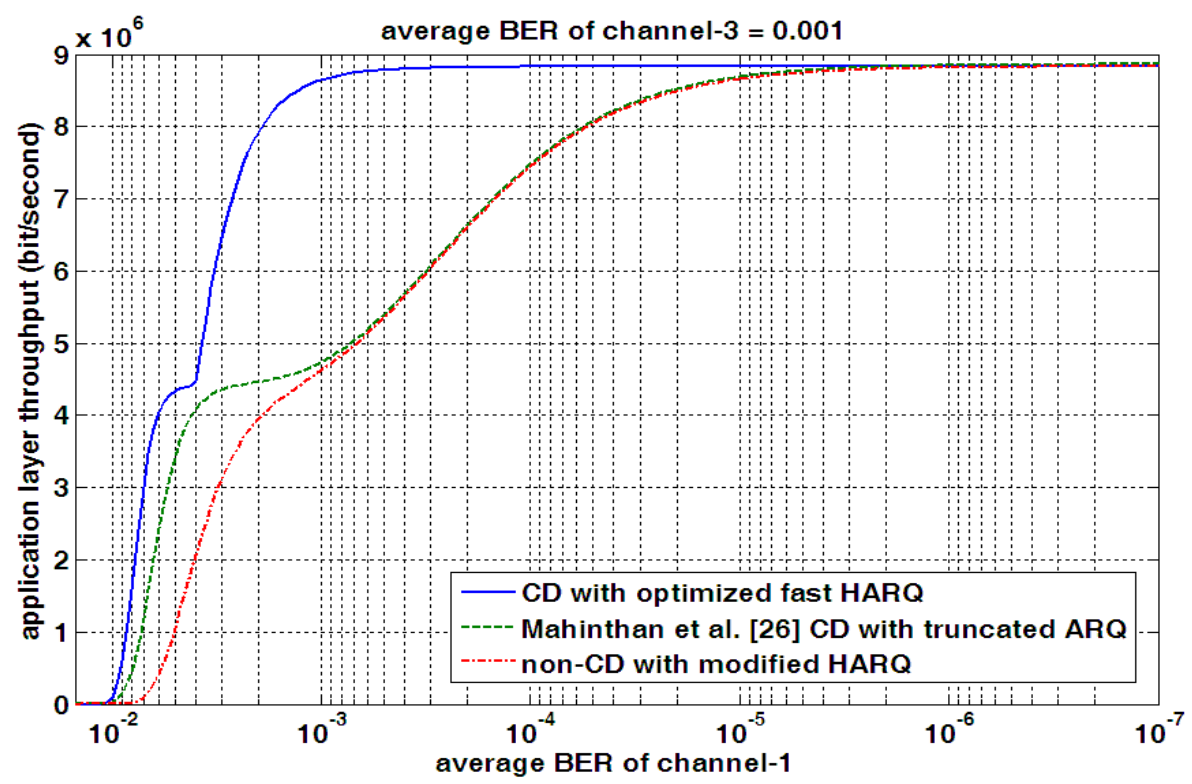

Fig. 6. Application layer throughput (in bit/sec.) comparison among various schemes under Nakagami-3 slow-fading channels when channel-2 is error-free.

Notice that, generally speaking, $\mathrm{BER}=0.001$ is fairly high (in our parameter setting, which is about equal to the packet error rate $=0.9$ when without employing any error correcting mechanism), that is to say, the channel condition is extremely bad. Here, the reasons for setting channel-3's BER $=0.001$ are explained as follows. Although the sender would like to select the neighboring partner having good channel condition for helping transmission, in the worst case when the partner is far away from the receiver and both of them are at the edge of a cell such that the channel-3's condition degrades. In this case, with the validation of analytical and simulation results, the performance result of our scheme is much better than that of other schemes. It means our scheme is very powerful. Thus, it can be easily 
reasoned that when channel-3's BER decreases, our scheme still remains the best although the performance results for these schemes will all be improved.

Furthermore, taking $\bar{\varepsilon}_{1}=2 \times 10^{-3}$ and $\bar{\varepsilon}_{3}=10^{-3}$ as an example, effective throughput performances of these schemes under different Nakagami- $m, m=1 / 2,1,3$, slow-fading channels in the CD environment are compared, as listed in Table 1. From Table 1, both analytical results based on (8)-(9) and simulation results show that the optimized fast HARQ scheme always achieves better throughput performance than other schemes since the optimized fast HARQ scheme can adaptively adjust the retransmission policy according to the channel quality. Again in Table 1, it is found that the analytical results are slightly lower than the simulation ones for both the optimized fast HARQ scheme and the non-CD HARQ scheme since the Viterbi decoding mechanism via (5) is employed for them. Note that the upper bound in (5) is tight and can be regarded as an excellent approximation when the BER is lower than $10^{-2}$ [36].

\begin{tabular}{|l|c|c|c|c|}
\hline \multicolumn{1}{|c|}{$\begin{array}{c}\text { Scheme } \\
\text { Environment }\end{array}$} & $\begin{array}{c}\text { Effective } \\
\text { Throughput } \\
\text { optimized } \\
\times 10^{6}\end{array}$ & $\begin{array}{c}\text { [26] CD with } \\
\text { fast HARQ }\end{array}$ & $\begin{array}{c}\text { Non-CD with } \\
\text { ARQ } \\
\text { modified } \\
\text { HARQ }\end{array}$ \\
\hline \multirow{2}{*}{ Nakagami-1/2 } & analytical result & 7.765 & 4.308 & 3.704 \\
\cline { 2 - 5 } & simulation result & 7.853 & 4.356 & 3.767 \\
\hline \multirow{2}{*}{ Nakagami-1 } & analytical result & 7.889 & 4.413 & 3.822 \\
\cline { 2 - 5 } & simulation result & 7.968 & 4.462 & 3.887 \\
\hline \multirow{2}{*}{ Nakagami-3 } & analytical result & 7.997 & 4.512 & 3.998 \\
\cline { 2 - 5 } & simulation result & 8.077 & 4.567 & 4.068 \\
\hline
\end{tabular}

Table 1. Comparisons of the application layer throughputs (in bit/second) at $\bar{\varepsilon}_{1}=2 \times 10^{-3}$ and $\bar{\varepsilon}_{3}=10^{-3}$ among various schemes under different Nakagami- $m, m=1 / 2,1,3$, slowfading channels.

\subsubsection{With a non-error-free channel-2}

Due to the fundamental physical characteristics of wireless channels, there often exists an error probability for each transmission channel in the real-world environment. However, in order to take the advantage of $\mathrm{CD}$, the sender usually selects the neighboring partner having good channel condition between them. Thus, we herein set $\bar{\varepsilon}_{2}=10^{-4}$ for demonstrating performance results. The throughput comparisons of various schemes under $\bar{\varepsilon}_{1}$, with $\bar{\varepsilon}_{2}=10^{-4}$ and $\bar{\varepsilon}_{3}=10^{-3}$ in Nakagami-3 slow-fading channels, are shown in Fig. 7.

It is found in Fig. 7 that the performance of the optimized fast HARQ scheme obviously degrades when the BER of channel-1 is smaller than $3 \times 10^{-3}$ when compared with that in Fig. 6. Because there exists an error probability on channel-2 and policy_0 only uses the cooperative path (i.e., channel-2 together with channel-3) for retransmissions, the power of policy_0 decreases. However, the throughput result of the optimized fast HARQ scheme in Fig. 7 is also shown better than that of the other 2 schemes. In addition, it can be observed 
that when $\bar{\varepsilon}_{1} \geq 3 \times 10^{-3}$, the performance results of the first 2 good schemes are almost the same as those in Fig. 6 due to the fact that MRC is much powerful.

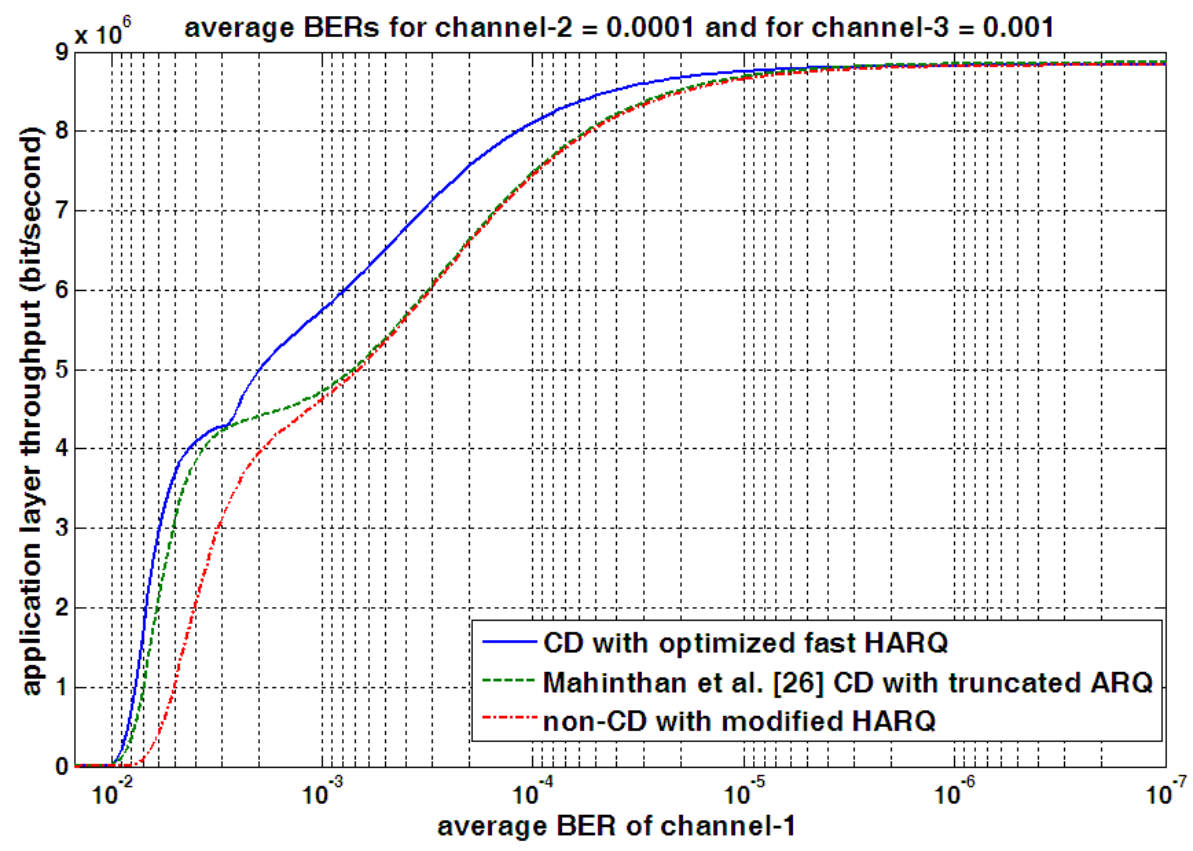

Fig. 7. Application layer throughput (in bit/sec.) comparison among various schemes under Nakagami-3 slow-fading channels when channel-2 is not error-free.

Last, for completeness, we take $\bar{\varepsilon}_{1}=2 \times 10^{-3}, \bar{\varepsilon}_{2}=10^{-4}$, and $\bar{\varepsilon}_{3}=10^{-3}$, as an example, to illustrate the effective throughput results for various schemes under different Nakagami- $m$, $m=1 / 2,1,3$, slow-fading channels, and summarize the results in Table 2 . From Table 2, it can be found that both analytical results based on (8)-(9) and simulation results of the optimized fast HARQ scheme also always have better throughput results than those of other schemes as in Table1. The results of the non-CD HARQ scheme for both Table 1 and Table 2 are the same since its performance only depends on channel-1's BER. We also notice that throughput improvement of our scheme is significant even with $\bar{\varepsilon}_{2}=10^{-4}$ in the sender-topartner channel.

In summary, based on Figs. 6 and 7 and Tables 1 and 2, we can thus conclude that the fast HARQ scheme is an excellent approach for transporting delay-constrained streaming-type or real-time multimedia flows in CD environments even when there is an error probability on the cooperative path. It is for the reasons that the retransmission strategy can be adaptively adjusted according to the channel condition and that the decoding procedure involving MRC and the Viterbi decoding are appropriately designed. 


\begin{tabular}{|c|c|c|c|c|}
\hline Environment & $\begin{array}{c}\text { Scheme } \\
\text { Effective } \\
\text { Throughput } \underset{\times 10^{6}}{ }\end{array}$ & $\begin{array}{c}\text { CD with } \\
\text { optimized } \\
\text { fast HARQ }\end{array}$ & $\begin{array}{c}\text { [26] CD with } \\
\text { truncated } \\
\text { ARQ }\end{array}$ & $\begin{array}{c}\text { Non-CD with } \\
\text { modified } \\
\text { HARQ }\end{array}$ \\
\hline \multirow[t]{2}{*}{ Nakagami-1/2 } & analytical result & 4.805 & 4.258 & 3.704 \\
\hline & simulation result & 4.848 & 4.258 & 3.767 \\
\hline \multirow[t]{2}{*}{ Nakagami-1 } & analytical result & 4.902 & 4.351 & 3.822 \\
\hline & simulation result & 4.951 & 4.351 & 3.887 \\
\hline \multirow[t]{2}{*}{ Nakagami-3 } & analytical result & 5.017 & 4.456 & 3.998 \\
\hline & simulation result & 5.067 & 4.456 & 4.068 \\
\hline
\end{tabular}

Table 2. Comparisons of the application layer throughputs (in bit/second) at $\bar{\varepsilon}_{1}=2 \times 10^{-3}$, $\bar{\varepsilon}_{2}=10^{-4}, \bar{\varepsilon}_{3}=10^{-3}$ among various schemes under different Nakagami- $m, m=1 / 2,1,3$, slow-fading channels.

\section{Conclusions}

A fast HARQ packet retransmission scheme has been successfully proposed to transport delay-sensitive flows in a general CD environment. The presented scheme incorporates 2 retransmission policies, and these 2 policies can be selected adaptively by the channel SNRs and the APDU sizes. In ideal conditions, the best retransmission policy can always be selected to achieve optimized performance.

In this case study, our cooperative fast retransmission scheme has been shown to be an excellent approach for improving the effective throughput in transporting delay-sensitive flows in CD environments. Numerical results verified via simulations, show that when optimized, the proposed scheme can achieve effective throughput much better than that of other ARQ schemes (such as [26]) and non-CD HARQ schemes, especially when the senderto-partner channel condition is good. The performance improvement is still significant even when there is an error probability (e.g. BER $\leq 10^{-4}$ ) in the sender-to-partner channel. Moreover, in the aspect of the battery saving, the presented scheme should save much more power than that of other schemes due to the one-time retransmission design. It is thus concluded that the proposed fast HARQ retransmission scheme is an excellent ARQ candidate for the multimedia or real-time transport in CD environments, when the timeconstraint is imposed.

\section{Summaries and future works}

The issues of improving fast retransmission schemes under $C D$ environments can be essential to many delay-sensitive applications. This chapter has widely covered the conceptual description of many representative retransmission schemes under various environments and presented a novel fast packet retransmission scheme intended for effectively transporting delay-sensitive flows in a general CD environment. The presented retransmission scheme and other related works should have provided a sufficient collection of schemes and analysis methodologies for designing further wireless communication systems with similar requirements. 
Furthermore, due to the fact that in most practical scenarios the terminals are batterypowered, the design of the energy-efficiency transmission satisfying the respectively specific QoS requirements of these users in the network is very crucial to prolonging the battery life of these terminals. Consequently, the issue concerning the energy consumption has been increasingly paid much attention. We suggest incorporating such a concern with the present work to design an efficiently power-saving fast packet retransmission scheme in a general $\mathrm{CD}$ environment for delay-sensitive flows in the future. Additionally, the well design of an efficient retransmission scheme to be employed in such a CD environment simultaneously considering the issue of effective throughput, QoS, fairness, complexity, and power saving is still an open issue for research.

\section{Acknowledgements}

The authors wish to express their sincere appreciation for financial support from the National Science Council of the Republic of China under Contracts NSC 98-2219-E-002-002 and NSC 99-2219-E-002-002.

\section{References}

[1] V. Mahinthan, J. W. Mark, and X. Shen, "A cooperative diversity scheme based on quadrature signaling," IEEE Trans. Wireless Commun., vol. 6, no. 1, pp. 41-45, January 2007.

[2] V. Mahinthan et al., "Maximizing cooperative diversity energy gain for wireless networks," IEEE Trans. Wireless Commun., vol. 6, no. 7, pp. 2530-2539, July 2007.

[3] V. Mahinthan et al., "Partner selection based on optimal power allocation in cooperative diversity systems," IEEE Trans. Veh. Technol., vol. 57, no. 1, pp. 511-520, January 2008.

[4] J. Chen and K. Djouani, "A multi-user cooperative diversity for wireless local area networks," Int. J. Communications, Network and System Sciences, vol. 3, pp. 207-283, 2008.

[5] K. J. Ray Liu, A. K. Sadek, W. Su, and A. Kwasinski, Cooperative communication and networking, Cambridge University Press, 2009.

[6] F. H. P. Fitzek and M. D. Katz, Cooperation in wireless networks: principles and applications, Springer, Netherlands, 2006.

[7] W. M. Jang, "Quantifying performance of cooperative diversity using the sampling property of a delta function," IEEE Trans. Wireless Commun., vol. 10, no. 7, pp. 20342039, July 2011.

[8] S. Lin, et al., “Automatic-repeat-request error control schemes," IEEE Commun. Mag., pp. 5-16, December 1984.

[9] J. So and N. H. Vaidya, "Multi-channel MAC for ad hoc networks: handling multichannel hidden terminals using a single transceiver," in Proc. ACM MobiHoc, pp. 222-233, Roppongi Hills, Tokyo, Japan, May 2004.

[10] R. Vedantham et al., "Component based channel assignment in single-radio multichannel ad hoc networks," in Proc. ACM MobiCom, pp. 378-389, Los Angeles, CA, USA, May 2006.

[11] W. H. Tam and Y. C. Tseng, "Joint multi-channel link layer and multi-path routing design for wireless mesh networks," in Proc. IEEE INFOCOM, pp. 2081-2089, May 2007. 
[12] P. Kyasanur and N. H. Vaidya, "Routing and link-layer protocols for multi-channel multi-interface ad hoc wireless networks," ACM Mobile Computing and Comm. Rev, pp. 31-43, vol. 10, no.1, January 2006.

[13] A. K. Jeng and R. H. Jan, "Optimization on hybrid channel assignment for multi-channel multi-radio wireless mesh networks," in Proc. IEEE GLOBECOM, November 2007.

[14] H. Su and X. Zhang, "Modeling throughput gain of network coding in multi-channel multi-radio wireless ad hoc networks," IEEE Journal on Selected Areas in Commun., vol. 27, no. 5, June 2009.

[15] N. Shacham and B. C. Shin, "A selective-repeat-ARQ protocol for parallel channels and its resequencing analysis," IEEE Trans. Commun., vol. COM-40, pp. 773-782, April 1992.

[16] J. F. Chang and T. H. Yang, "Multichannel ARQ protocols," IEEE Trans. Commun., vol. COM-41, pp. 592-598, April 1993.

[17] Z. Ding and M. Rice, "ARQ error control for parallel multichannel communications," IEEE Trans. Wireless Commun., vol. COM-5, no. 11, pp. 3039-3044, November 2006.

[18] Y.-L. Chung and Z. Tsai, "Performance analysis of two multichannel fast retransmission schemes for delay-sensitive flows," IEEE Trans. Veh. Technol., vol. 59, no. 7, pp. 3468-3479, September 2010.

[19] W. Luo, K. Balachandran, S. Nanda, and K. K. Chang, "Delay analysis of selectiverepeat ARQ with applications to link adaptation in wireless packet data systems," IEEE Trans. Wireless Commun., vol. 4, no. 3, pp. 1017-1029, May 2005.

[20] L. Badia, M. Rossi, and M. Zorzi, "SR ARQ packet delay statistics on Markov channels in the presence of variable arrival rate," IEEE Trans. Wireless Commun., vol. 5, no. 7, pp. 1639-1644, July 2006.

[21] L. B. Le, E. Hossain, and M. Zorzi, "Queueing analysis for GBN and SR ARQ protocols under dynamic radio link adaptation with non-zero feedback delay," IEEE Trans. Wireless Commun., vol. 6, no. 9, pp. 3418-3428, September 2007.

[22] L. Badia, M. Levorato, and M. Zorzi, "Markov analysis of selective repeat type II hybrid ARQ using block codes," IEEE Trans. Commun., vol. 56, no. 9, pp. 1434-1441, September 2008.

[23] T. V. K. Chaitanya and E. G. Larsson, “Outage-optimal power allocation for hybrid ARQ with incremental redundancy," IEEE Trans. Wireless Commun., vol. 10, no. 7, pp. 2069-2074, July 2011.

[24] H. Boujemâa, "Delay analysis of cooperative truncated HARQ with opportunistic relaying," IEEE Trans. Veh. Technol., vol. 58, no. 9, pp. 4795-4803, November 2009.

[25] L. Le and E. Hossain, "An analytical model for ARQ cooperative diversity in multi-hop wireless networks," IEEE Trans. Wireless Commun., vol. 7, no. 5, pp. , 1786-1791, May 2008.

[26] V. Mahinthan et al., "Cross-layer performance study of cooperative diversity system with ARQ," IEEE Trans. Veh. Technol., vol. 58, no. 2, pp. 705-719, February 2009.

[27] T. Issariyakul, and V. Krishnamurthy, "Amplify-and-forward cooperative diversity wireless networks: models, analysis, and monotonicity properties," IEEE/ACM Trans. Networking, vol. 17, no. 1, pp. 225-238, February 2009.

[28] M. Nakagami, "The m-distribution - a general formula of intensity distribution of rapid fading," in Statistical Methods of Radio Wave Propagation, pp. 3-36, W. C. Hoffman Ed. Elmsford, Pergamon Press, New York, 1960. 
[29] L. R. Lugand, et al., "Parity retransmission hybrid ARQ using rate- $1 / 2$ convolutional codes on a nonstationary channel," IEEE Trans. Commun., Vol. 37, no. 7, pp. 755765, July 1989.

[30] M. S. Patterh, et al., "BER performance of MQAM with L-branch MRC diversity reception over correlated Nakagami-m fading channels," International Journal of Wireless Communications and Mobile Computing, vol. 3, pp. 397-406, May 2003.

[31] D. G. Brennan, "Linear diversity combining techniques," Proc. IRE, vol. 47, pp. 10751102, June 1959.

[32] D. Yoon, K. Cho, and J. Lee, "Bit error probability of M-ary quadrature amplitude modulation," in Proc. the IEEE VTC, Boston, MA, September 2000.

[33] Y.-L. Chung and Z. Tsai, "On the effective throughput gain of cooperative diversity with a fast retransmission scheme for delay-sensitive flows," IEICE Trans. Commun., vol. E94-B, no.12, pp.-, December 2011.

[34] Y.-L. Chung and Z. Tsai, "Cooperative diversity with fast HARQ for delay-sensitive flows," in Proc. IEEE 71st Veh. Technol. Conf. (IEEE VTC), Taipei, Taiwan, May 2010.

[35] A. Leon-Garcia, Probability, statistics, and random processes for electrical engineering, 3rd ed., Prentice Hall, 2008.

[36] A. J. Viterbi and J. K. Omura, Principles of digital communication and coding, McGrawHall, 1979. 


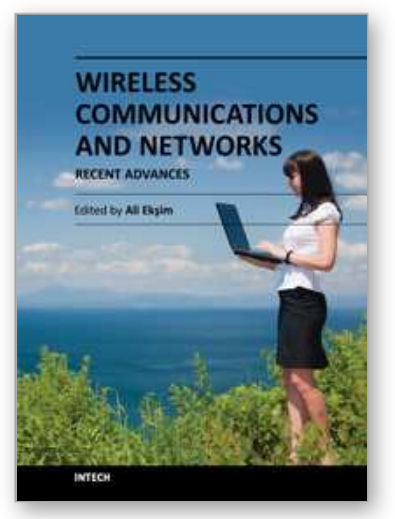

\author{
Wireless Communications and Networks - Recent Advances \\ Edited by Dr. Ali Eksim
}

ISBN 978-953-51-0189-5

Hard cover, 596 pages

Publisher InTech

Published online 14, March, 2012

Published in print edition March, 2012

This book will provide a comprehensive technical guide covering fundamentals, recent advances and open issues in wireless communications and networks to the readers. The objective of the book is to serve as a valuable reference for students, educators, scientists, faculty members, researchers, engineers and research strategists in these rapidly evolving fields and to encourage them to actively explore these broad, exciting and rapidly evolving research areas.

\title{
How to reference
}

In order to correctly reference this scholarly work, feel free to copy and paste the following:

Yao-Liang Chung and Zsehong Tsai (2012). Introduction to the Retransmission Scheme Under Cooperative Diversity in Wireless Networks, Wireless Communications and Networks - Recent Advances, Dr. Ali Eksim (Ed.), ISBN: 978-953-51-0189-5, InTech, Available from: http://www.intechopen.com/books/wirelesscommunications-and-networks-recent-advances/introduction-to-the-retransmission-scheme-undercooperative-diversity-in-wireless-networks

\section{INTECH}

open science | open minds

\section{InTech Europe}

University Campus STeP Ri

Slavka Krautzeka 83/A

51000 Rijeka, Croatia

Phone: +385 (51) 770447

Fax: +385 (51) 686166

www.intechopen.com

\section{InTech China}

Unit 405, Office Block, Hotel Equatorial Shanghai

No.65, Yan An Road (West), Shanghai, 200040, China

中国上海市延安西路65号上海国际贵都大饭店办公楼 405 单元

Phone: +86-21-62489820

Fax: +86-21-62489821 
(C) 2012 The Author(s). Licensee IntechOpen. This is an open access article distributed under the terms of the Creative Commons Attribution 3.0 License, which permits unrestricted use, distribution, and reproduction in any medium, provided the original work is properly cited. 